International Journal of Applied Mathematics

Volume 34 No. $6 \quad 2021,1093-1109$

ISSN: 1311-1728 (printed version); ISSN: 1314-8060 (on-line version)

doi: http://dx.doi.org/10.12732/ijam.v34i6.4

\title{
OPTIMAL CONTROL OF AN HIV INFECTION MODEL WITH THE CTL RESPONSE AND TWO SATURATED RATES
}

\author{
Bouchra Lahmidani ${ }^{1}$, Othmane Baiz ${ }^{2}$, \\ Youssef Tabit $^{3}$, Driss El Moutawakil ${ }^{4}$ \\ ${ }^{1}$ Univ. Sultan Moulay Slimane, Equipe de recherche MATIC \\ FPK, MOROCCO \\ ${ }^{2}$ Univ. Ibn Zohr, FP of Ouarzazate, MOROCCO \\ ${ }^{3}$ Univ. Hassan II, ENCG Casablanca, MOROCCO \\ ${ }^{4}$ Univ. Sultan Moulay Slimane, Equipe de recherche MATIC \\ FPK, MOROCCO
}

\begin{abstract}
In this paper, we study a mathematical model that describes the Human Immunodeficiency Virus (HIV) pathogenesis with Cytotoxic T Lymphocytes (CTL) response. This model includes the cure of infected cells, two saturated rates describing viral infection, CTL proliferation and two treatments. These latter represent the efficiency of drug treatment in inhibiting viral production and preventing new infections. First, we derive the positivity and boundedness of solutions for nonnegative initial data, which is consistent with the biological studies. Furthermore, we prove the existence of the optimal control pair, and its characterization is obtained by using the Pontryagin's maximum principle. Finally, the optimality system is derived and illustrated by a numerical example. The obtained results show that the optimal treatment strategies reduce the viral load and then increase the uninfected $C D 4^{+} \mathrm{T}$ cells as well as cytotoxic T-lymphocyte immune response, this improves the patient life quality.
\end{abstract}

AMS Subject Classification: 92Bxx, 35Fxx, 49Kxx, 49Lxx, 49J15 Key Words: HIV; immune response; treatment; optimal control; Pontryagin's principle

Received: February 10, 2021

(c) 2021 Academic Publications

$\S_{\text {Correspondence author }}$ 


\section{Introduction}

It is well known that the Human Immunodeficiency Virus (HIV) is a pathogen that causes well-known Acquired Immunodeficiency Syndrome (AIDS). After this resulted end-stage of the viral infection, the immune system fails to play its role $[1,2]$. Currently, there is no cure or vaccine for HIV [3]. However, antiretroviral (ART) treatment is used to treat HIV. For the antiretroviral, there are two kinds which are medications licensed for the treatment of infected individuals with HIV: reverse transcriptase inhibitors (RTIs) and protease inhibitors (PIs) for more details see for example [4]. The reverse transcriptase inhibitors (RTIs) oppose the conversion of RNA of the virus to DNA (reverse transcription), consequently, the viral population will be minimum and the $\mathrm{CD} 4^{+} \mathrm{T}$ cells count remains higher. The protease inhibitors (PIs) prevents the production of viruses from the actively infected $\mathrm{CD} 4^{+} \mathrm{T}$ cells. Some general models and their analysis have been established for the dynamics of HIV infection include $[5,6,7,8,9,10,11,12,13,14,15,16,17]$.

One of the earliest of these models was the basic virus infection model introduced by Nowak and al. [6, 10] and Perelson and al. [7, 9]. Nowak et al. [6] extended the basic model

$$
\left\{\begin{array}{l}
\dot{x}(t)=\lambda-d x-\beta v x, \\
\dot{y}(t)=\beta v x-\delta y, \\
\dot{v}(t)=k y-\mu v,
\end{array}\right.
$$

to include the population of CTL cells, and the model became as follows:

$$
\left\{\begin{array}{l}
\dot{x}(t)=\lambda-d x-\beta v x, \\
\dot{y}(t)=\beta v x-\delta y-p y z, \\
\dot{v}(t)=k y-\mu v, \\
\dot{z}(t)=c y z-b z .
\end{array}\right.
$$

Here $x, y, v$, and $z$ denote the uninfected cells, infected cells, free virus and Cytotoxic T Lymphocytes(CTL) cells, respectively. The susceptible host cells $\mathrm{CD}^{+} \mathrm{T}$ cells are produced at a rate $\lambda$, die at a rate $d x$ and become infected by the virus at a rate $\beta v x$. Infected cells die at a rate $\delta y$ and are killed by the CTL response at a rate pyz. Free virus $(v)$ is produced by infected cells at a rate $k y$, decays at a rate $\mu v$. CTLs expand in response to a viral antigen derived from infected cells at a rate $c y z$ and decay in the absence of antigenic stimulation at a rate $b z$. The stability analysis of the model (2) is made by Liu 
in [8]. Ciupe et al. [11] assumed that the production of CTL depends only on the concentration of infected cells, as presented in the following model:

$$
\left\{\begin{array}{l}
\dot{x}(t)=\lambda-d x-\beta v x \\
\dot{y}(t)=\beta v x-\delta y-p y z \\
\dot{v}(t)=N \delta y-\mu v \\
\dot{z}(t)=q y-b z
\end{array}\right.
$$

where $N$ is the number of virus particles produced by infected cells and $q$ is the proliferation rate of CTLs. K. Hattaf et al. [12] introduced two drugs in the basic model, reverse transcriptase inhibitors (RTIs) and protease inhibitors (PIs), and they introduced the cure rate of infected cells to the susceptible host cells due to the noncytolytic processes $[18,19]$. They included two types of virus particles to model (1). The virus not being influenced by protease inhibitors PIs, $\left(v_{I}\right)$, and the other, $\left(v_{N I}\right)$, being influenced by PIs. They obtained the following model:

$$
\left\{\begin{aligned}
\dot{x}(t) & =\lambda-d x-(1-\eta) \beta v_{I} x+r y \\
\dot{y}(t) & =(1-\eta) \beta v_{I} x-(\delta+r) y, \\
\dot{v}_{I}(t) & =(1-\epsilon) k y-\mu v_{I}, \\
\dot{v}_{N I}(t) & =\epsilon k y-\mu v_{N I},
\end{aligned}\right.
$$

where $r$ is the cure rate of the infected cells $y$. The new parameters $\eta$ and $\epsilon$ measure the efficacy of reverse transcriptase inhibitor and protease inhibitor respectively. El boukhari and al. [13] extended model (4) to include the population of CTL cells, and they obtained the following model:

$$
\left\{\begin{aligned}
\dot{x}(t) & =\lambda-d x-(1-\eta) \beta v_{I} x+r y, \\
\dot{y}(t) & =(1-\eta) \beta v_{I} x-(\delta+r) y-p y z \\
\dot{v}_{I}(t) & =(1-\epsilon) k y-\mu v_{I}, \\
\dot{v}_{N I}(t) & =\epsilon k y-\mu v_{N I}, \\
\dot{z}(t) & =c y z-b z
\end{aligned}\right.
$$

with initial conditions $x(0)=x_{0}, y(0)=y_{0}, v_{I}(0)=v_{I 0}, v_{N I}(0)=v_{N I 0}$ and $z(0)=z_{0}$. Note that the rate of infection in Eq. (5) is assumed to be bilinear in the virus $v_{I}$ and the uninfected target cells $x$, which is not reasonable to describe the HIV infection. Hence, we replace this bilinear form with a saturated mass action presented by Song and al. [20]. Furthermore, the proliferation of CTL response in Eq. (5) is bilinear in $y$ and $z$. However, in the presence of immune 
impairment effects caused by HIV infection, CTL proliferation is reduced (see [21]). Moreover, in several works the constants $\eta$ and $\epsilon$ are taken as variables, see for examples $[22,23,24,25]$. These reasons allow us to extend the model Eq. (5) to the following model:

$$
\left\{\begin{aligned}
\dot{x}(t) & =\lambda-d x(t)-\left(1-u_{1}(t)\right) \frac{\beta v_{I} x}{1+a v_{I}}+r y, \\
\dot{y}(t) & =\left(1-u_{1}(t)\right) \frac{\beta v_{I} x}{1+a v_{I}}-(\delta+r) y-p y z \\
\dot{v}_{I}(t) & =\left(1-u_{2}(t)\right) k y-\mu v_{I} \\
\dot{v}_{N I}(t) & =u_{2}(t) k y-\mu v_{N I} \\
\dot{z}(t) & =\frac{c y z}{1+\alpha y}-b z
\end{aligned}\right.
$$

where $a$ is the saturation response of the infection rate and $\alpha$ describes the immune impairment rate, and $u=\left(u_{1}(t), u_{2}(t)\right)$ represents an antiviral therapy time dependent. The first control $u_{1}(t)$ is the efficiency of drug therapy in blocking new infection; while the second one $u_{2}(t)$ stands for the efficiency of drug therapy in inhibiting viral production. The purpose of this paper is to find an optimal control that will minimize the viral load and maximize healthy cells using similar optimal control techniques.

The rest of the paper is organized as follows. In Section 2, we state and prove the existence, positivity, and boundedness of solutions. An optimal control problem introduced and analyzed based on the Pontryagin maximum principle in Section 3. In Section 4, we construct an appropriate numerical algorithm, we report computer simulation results that provide numerical evidence of the numerical algorithm, and give a discussion of the results for the optimal control problem.

\section{Positivity and boundedness of solutions}

In this section, we start by presenting our existence result of Eq. (6). We recall that the model that we want to analyze describes the evolution of a cell population. Hence, the cell densities should remain non-negative and bounded. For biological reasons, we assume that the parameters $x_{0}, v_{I 0}, v_{N I 0}$ and $z_{0}$ are larger than or equal to 0 . Then, We have the following result.

Theorem 1. The problem (6) has at least one solution. Moreover, the solutions of problem (6) are bounded, nonnegative and verify: 
(i) $x_{1}(t) \leq x_{1}(0)+\frac{\lambda}{\delta_{1}}$,

(ii) $v_{I}(t) \leq v_{I}(0)+\frac{k}{\mu}\|y\|_{L^{\infty}}$,

(iii) $v_{N I}(t) \leq v_{N I}(0)+\frac{k}{\mu}\|y\|_{L^{\infty}}$,

(iv) $z(t) \leq z(0)+\frac{c}{p} y(t)-\frac{c}{p} y(0)+\frac{c}{p}(\delta+r)\|y\|_{\infty}$,

where $x_{1}(t)=x(t)+y(t)$ and $\delta_{1}=\min (d ; \delta)$.

Proof. First, we show that the nonnegative orthant

$$
\mathbb{R}_{+}^{5}=\left\{\left(x, y, v_{I}, v_{N I}, z\right) \in \mathbb{R}^{5}: x \geq 0, y \geq 0, v_{I} \geq 0, v_{N I} \geq 0 \text { and } z \geq 0\right\}
$$

is positively invariant. Indeed, for $\left(x(t), y(t), v_{I}(t), v_{N I}(t), z(t)\right) \in \mathbb{R}_{+}^{5}$ we have,

$$
\left\{\begin{array}{l}
\left.\dot{x}\right|_{x=0}=\lambda+r y \geq 0 \\
\left.\dot{y}\right|_{y=0}=\frac{\left(1-u_{1}\right) \beta v_{I} x}{1+a v_{I}} \geq 0 \\
\left.\dot{v}_{I}\right|_{v=0}=\left(1-u_{2}\right) k y \geq 0 \\
\left.\dot{v}_{N I}\right|_{v_{N I}=0}=u_{2} k y \geq 0 \\
\left.\dot{z}\right|_{z=0}=0 \geq 0
\end{array}\right.
$$

Therefore, all solutions initiating in $\mathbb{R}_{+}^{5}$ are positive.

Next, we will show that these solutions remain bounded. For that, we begin by adding the first and second equations in (6), to get

$$
\dot{x}_{1}=\lambda-d x-\delta y-p y z,
$$

thus

$$
x_{1}(t) \leq x_{1}(0) e^{-\delta_{1} t}+\frac{\lambda}{\delta_{1}}\left(1-e^{-\delta_{1} t}\right),
$$

where $\delta_{1}=\min (d ; \delta)$. Since $0 \leq e^{-\delta_{1} t} \leq 1$ and $1-e^{-\delta_{1} t} \leq 1$, we deduce $(i)$. This proves that $x$ and $y$ are bounded.

From the equation $\dot{v}_{I}=\left(1-u_{2}(t)\right) k y(t)-\mu v_{I}$, we obtain

$$
v_{I}(t)=v_{I}(0) e^{-\mu t}+k \int_{0}^{t}\left(1-u_{2}(t)\right) y(\xi) e^{(\xi-t) \mu} d \xi
$$


Since $0 \leq\left(1-u_{2}(t)\right) \leq 1$, then

$$
v_{I}(t) \leq v_{I}(0)+\frac{k}{\mu}\|y\|_{\infty}\left(1-e^{-\mu t}\right) .
$$

The fact that $0 \leq 1-e^{-\mu t} \leq 1$ implies that $(i i)$ is verified.

Now, from the equation $v_{\dot{N}_{I}}=u_{2}(t) k y(t)-\mu v_{N I}$, we get

$$
v_{N I}(t)=v_{N I}(0) e^{-\mu t}+k \int_{0}^{t} u_{2}(t) y(\xi) e^{(\xi-t) \mu} d \xi .
$$

Since $\left.0 \leq u_{2}(t)\right) \leq 1$, we have

$$
v_{N I}(t) \leq v_{N I}(0)+\frac{k}{\mu}\|y\|_{\infty}\left(1-e^{-\mu t}\right) .
$$

Note that $0 \leq 1-e^{-\mu t} \leq 1$, then, we deduce $($ iii $)$.

Next, we show $(i v)$. The equation $\dot{z}=\frac{c y z}{1+\alpha y}-b z$ implies that

$$
\dot{z}-b z \leq c y z \text {. }
$$

Since $c y z=\frac{c}{p}[\lambda-(\dot{x}+d x)+(\dot{y}+\delta y)]$, then

$$
\begin{array}{r}
z(t) \leq\left[\frac{c}{p}\left(x(0)+y(0)-\frac{\lambda}{b}\right)+z(0)\right] e^{-b t} \\
+\frac{c}{p}\left\{\frac{\lambda}{b}+\int_{0}^{t}[(b-d) x(\xi)+(b-\delta) y(\xi)] e^{b(\xi-t)} d \xi-x(t)-y(t)\right\} .
\end{array}
$$

$\diamond$ Case 1 : If $b-d \leq 0$ and $b-\delta \leq 0$, we have

$$
z(t) \leq z(0)+\frac{c}{p}\left[\frac{\lambda}{b}+x(0)+y(0)\right] .
$$

$\diamond$ Case 2 : If $b-d \leq 0$ and $b-\delta \geq 0$, we have

$$
z(t) \leq z(0)+\frac{c}{p}\left[\frac{\lambda}{b}+x(0)+y(0)+\left(1-\frac{\delta}{b}\right)\|y\|_{\infty}\right] .
$$

$\diamond$ Case 3 : If $b-d \geq 0$ and $b-\delta \leq 0$, we have

$$
z(t) \leq z(0)+\frac{c}{p}\left[\frac{\lambda}{d}+\left(2-\frac{d}{b}\right) x(0)+y(0)\right] .
$$

$\diamond$ Case $4:$ If $b-d \geq 0$ and $b-\delta \geq 0$, we have

$$
z(t) \leq z(0)+\frac{c}{p}\left[\frac{\lambda}{d}+\left(2-\frac{d}{b}\right) x(0)+y(0)+\left(1-\frac{\delta}{b}\right)\|y\|_{\infty}\right] .
$$

Therefore, we have proved that all the cases imply $(i v)$. 


\section{The optimal control problem}

We have seen in the previous section that for a given control function $u=$ $\left(u_{1}, u_{2}\right)$ the problem has a solution. The optimization problem under consideration is to maximize the following objective functional

$$
J\left(u_{1}, u_{2}\right)=\int_{0}^{t_{f}}\left\{x(t)+z(t)-\left[\frac{A_{1}}{2} u_{1}^{2}(t)+\frac{A_{2}}{2} u_{2}^{2}(t)\right]\right\} d t,
$$

where $t_{f}$ is the time period of treatment and the positive constants $A_{1}$ and $A_{2}$ stand for the benefits and costs of the introduced treatment. The two control functions, $u_{1}(t)$ and $u_{2}(t)$ are assumed to be bounded and Lebesgue integrable. Let $U$ be the control set defined by

$$
U=\left\{\left(u_{1}(t), u_{2}(t)\right): u_{i}(t) \text { measurable, } 0 \leq u_{i}(t) \leq 1, t \in\left[0, t_{f}\right], i=1,2\right\} .
$$

We consider the following optimal control problem: Find $\left(u_{1}^{*}, u_{2}^{*}\right) \in U$ such that,

$$
J\left(u_{1}^{*}, u_{2}^{*}\right)=\max \left\{J\left(u_{1}, u_{2}\right):\left(u_{1}, u_{2}\right) \in U\right\} .
$$

We are now in a position to deliver an existence result for the optimal control pair problem, which is a direct consequence of the results found in [27, 28].

Theorem 2. There exists an optimal control pair $\left(u_{1}^{*}, u_{2}^{*}\right) \in U$ such that

$$
J\left(u_{1}^{*}, u_{2}^{*}\right)=\max _{\left(u_{1}, u_{2}\right) \in U} J\left(u_{1}, u_{2}\right) .
$$

Proof. In order to use the existence result proved in [27], we must check the following properties:

$\left(P_{1}\right)$ The set of controls and corresponding state variables is nonempty.

$\left(P_{2}\right)$ The control $U$ set is convex and closed.

$\left(P_{3}\right)$ The right hand side of the state system is bounded by a linear function of the state and control variables.

$\left(P_{4}\right)$ The integrand of the objective functional is concave on $U$.

$\left(P_{5}\right)$ There exists constants $c_{1}, c_{2}>0$, and $\beta>1$ such that the integrand of the objective functional satisfies

$$
L\left(x, z, u_{1}, u_{2}\right) \leq c_{2}-c_{1}\left(\left|u_{1}\right|^{2}+\left|u_{2}\right|^{2}\right)^{\frac{\beta}{2}} .
$$


Using the result proved by Lukes in [28], we show the existence of solutions of system $(6)$, which gives the condition $\left(P_{1}\right)$. It follows from the definition of the control set that the set $U$ satisfies $\left(P_{2}\right)$. Furthermore, we can easily see that our state system is bilinear in $u_{1}, u_{2}$, using the boundedness of the solutions, then the right hand side of the system (6) satisfies condition $\left(P_{3}\right)$. Moreover, the integrand of our objective function is concave. Finally, we have

$$
L\left(x, z, u_{1}, u_{2}\right) \leq c_{2}-c_{1}\left(\left|u_{1}\right|^{2}+\left|u_{2}\right|^{2}\right),
$$

where $c_{2}$ depends on the upper bound on $T, W, Z$, and $c_{1}$ is positive constant depends only on $A_{1}>0$ and $A_{2}>0$. Hence, we are in a position to apply the result of existence in [28] to the optimal control (15). We deduce that exists an optimal control pair $\left(u_{1}^{*}, u_{2}^{*}\right) \in U$ such that

$$
J\left(u_{1}^{*}, u_{2}^{*}\right)=\max _{\left(u_{1}, u_{2}\right) \in U} J\left(u_{1}, u_{2}\right) .
$$

Next, we will provide the necessary conditions for the optimal control problem. Recall that Pontryagin's maximum principle given in [29] transforms (6), (13) and (14) into a problem of maximizing a Hamiltonian, $H$, pointwisely with respect to $u_{1}$ and $u_{2}$, formulated as follows,

$$
H\left(t, x, y, v_{I}, v_{N I}, z, u_{1}, u_{2}, \lambda\right)=\left(\frac{A_{1}}{2} u_{1}^{2}+\frac{A_{2}}{2} u_{2}^{2}\right)-x-z+\sum_{i=1}^{5} \lambda_{i} f_{i}
$$

where $f_{i}$ is the right hand side of the differential equation of $i$-th state variable, which is described as follows:

$$
\left\{\begin{array}{l}
f_{1}=\lambda(t)-d x(t)-\left(1-u_{1}(t)\right) \frac{\beta v_{I}(t) x(t)}{1+a v_{I}(t)}+r y(t) \\
f_{2}=\left(1-u_{1}(t)\right) \frac{\beta v_{I} x(t)}{1+a v_{I}(t)}-(\delta+r) y(t)-p y(t) z(t) \\
f_{3}=\left(1-u_{2}(t)\right) k y(t)-\mu v_{I} \\
f_{4}=u_{2}(t) k y(t)-\mu v_{N I}(t) \\
f_{5}=\frac{c y(t) z(t)}{1+\alpha y(t)}-b z(t)
\end{array}\right.
$$

We complete Theorem 1 and Theorem 2 with the following result. 
Theorem 3. For any optimal control $u_{1}^{*}, u_{2}^{*}$ and solutions $x^{*}, y^{*}, v_{I}^{*}, v_{N I}^{*}$ and $z^{*}$ of the corresponding state system (6), there exist adjoint variables, $\lambda_{1}, \lambda_{2}, \lambda_{3}, \lambda_{4}$ and $\lambda_{5}$, satisfying the equations

$$
\left\{\begin{array}{l}
\lambda_{1}^{\prime}(t)=1+\lambda_{1}(t) d+\left(\lambda_{1}(t)-\lambda_{2}(t)\right) \frac{\left(1-u_{1}^{*}(t)\right) \beta v_{I}^{*}(t)}{1+a v_{I}^{*}(t)} \\
\lambda_{2}^{\prime}(t)=-\lambda_{1}(t) r+\lambda_{2}(t)\left(\delta+r+p Z^{*}(t)\right) \\
\lambda_{3}(t)\left(1-u_{2}^{*}(t)\right) k-\lambda_{4} u_{2}^{*}(t) k-\lambda_{5}(t) \frac{c Z^{*}(t)}{\left(1+\alpha y^{*}(t)\right)^{2}} \\
\lambda_{3}^{\prime}(t)=\left(\lambda_{1}(t)-\lambda_{2}(t)\right)\left(1-u_{1}^{*}(t)\right) \frac{\beta x^{*}(t)}{\left(1+a v_{I}^{*}(t)\right)^{2}}+\lambda_{3}(t) \mu \\
\lambda_{4}^{\prime}(t)=\lambda_{4}(t) \mu \\
\lambda_{5}^{\prime}(t)=1+\lambda_{2}(t) p y^{*}(t)-\lambda_{5}(t)\left(\frac{c y^{*}(t)}{1+\alpha y^{*}(t)}-b\right)
\end{array}\right.
$$

with the transversality conditions

$$
\lambda_{i}\left(t_{f}\right)=0, \quad i=1, \ldots, 5
$$

Moreover, the optimal control is given by

$$
\left\{\begin{array}{l}
u_{1}^{*}(t)=\min \left(1, \max \left(0, \frac{1}{A_{1}}\left(\lambda_{2}-\lambda_{1}\right) \frac{\beta v_{I}^{*}(t) x^{*}(t)}{1+a v_{I}^{*}(t)}\right)\right), \\
u_{2}^{*}(t)=\min \left(1, \max \left(0, \frac{1}{A_{2}}\left(\lambda_{3}-\lambda_{4}\right) k y^{*}(t)\right)\right) .
\end{array}\right.
$$

Proof. The adjoint equations and transversality conditions can be obtained by using Pontryagin's Maximum Principal such that

$$
\begin{cases}\lambda_{1}^{\prime}(t)=-\frac{\partial H}{\partial x}(t), & \lambda_{1}\left(t_{f}\right)=0, \\ \lambda_{2}^{\prime}(t)=-\frac{\partial H}{\partial y}(t), & \lambda_{2}\left(t_{f}\right)=0, \\ \lambda_{3}^{\prime}(t)=-\frac{\partial H}{\partial v_{I}}(t), & \lambda_{3}\left(t_{f}\right)=0, \\ \lambda_{4}^{\prime}(t)=-\frac{\partial H}{\partial v_{N I}}(t), & \lambda_{4}\left(t_{f}\right)=0, \\ \lambda_{5}^{\prime}(t)=-\frac{\partial H}{\partial z}(t), & \lambda_{5}\left(t_{f}\right)=0 .\end{cases}
$$

The two optimal controls $u_{1}^{*}$ and $u_{2}^{*}$ can be solved from the optimality conditions

$$
\frac{\partial H}{\partial u_{1}}(t)=0, \quad \frac{\partial H}{\partial u_{2}}(t)=0 .
$$

That is, 


$$
\begin{aligned}
& \frac{\partial H}{\partial u_{1}}(t)=A_{1} u_{1}(t)+\left(\lambda_{1}^{*}-\lambda_{2}^{*}\right) \frac{\beta v_{I}^{*}(t) x^{*}(t)}{1+a v_{I}^{*}(t)}=0, \\
& \frac{\partial H}{\partial u_{2}}(t)=A_{2} u_{2}(t)-\left(\lambda_{3}^{*}-\lambda_{4}^{*}\right) k y^{*}(t)=0 .
\end{aligned}
$$

Finally, using the fact that $\left(u_{1}^{*}, u_{2}^{*}\right) \in U$, we find that $u_{1}^{*}$ and $u_{2}^{*}$ have the form (18).

The optimality system consists of the state system coupled with the adjoint system with the initial conditions, the transversality conditions, and the characterization of the optimal control. We substitute the forms of $u_{1}^{*}$ and $u_{2}^{*}$ in the system (6), to obtain the following optimality system:

$$
\begin{aligned}
& \frac{d x^{*}(t)}{d t}=\lambda(t)-d x^{*}(t)-\left(1-u_{1}\right) \frac{\beta v_{I}^{*}(t) x^{*}(t)}{1+a v_{I}^{*}(t)}+r y^{*}(t), \\
& \frac{d y^{*}(t)}{d t}=\left(1-u_{1}\right) \frac{\beta v_{I}^{*} x(t)}{1+a v_{I}^{*}(t)}-(\delta+r) y^{*}(t)-p y^{*}(t) z^{*}(t), \\
& \frac{d v_{I}^{*}(t)}{d t}=\left(1-u_{2}\right) k y^{*}(t)-\mu v_{I}^{*}, \\
& \frac{d v_{N I}^{*}(t)}{d t}=u_{2} k y^{*}(t)-\mu v_{N I}^{*}(t) \\
& \frac{d z^{*}(t)}{d t}=\frac{c y^{*}(t) z^{*}(t)}{1+\alpha y^{*}(t)}-b z^{*}(t), \\
& \frac{d \lambda_{1}(t)}{d t}=1+\lambda_{1}(t) d+\left(\lambda_{1}(t)-\lambda_{2}(t)\right) \frac{\left(1-u_{1}^{*}(t)\right) \beta v_{I}^{*}(t)}{1+a v_{I}^{*}(t)}, \\
& \frac{d \lambda_{2}(t)}{d t}=-\lambda_{1}(t) r+\lambda_{2}(t)\left(\delta+r+p Z^{*}(t)\right) \\
& -\lambda_{3}(t)\left(1-u_{2}^{*}(t)\right) k-\lambda_{4} u_{2}^{*}(t) k-\lambda_{5}(t) \frac{c Z^{*}(t)}{\left(1+\alpha y^{*}(t)\right)^{2}}, \\
& \frac{d \lambda_{3}(t)}{d t}=\left(\lambda_{1}(t)-\lambda_{2}^{*}(t)\right)\left(1-u_{1}^{*}(t)\right) \frac{\beta x^{*}(t)}{\left(1+a v_{I}^{*}(t)\right)^{2}}+\lambda_{3}^{*}(t) \mu, \\
& \frac{d \lambda_{4}(t)}{d t}=\lambda_{4}^{*}(t) \mu
\end{aligned}
$$




$$
\begin{gathered}
\frac{d \lambda_{5}(t)}{d t}=1+\lambda_{2}^{*}(t) p y^{*}(t)-\lambda_{5}^{*}(t)\left(\frac{c y^{*}(t)}{1+\lambda y^{*}(t)}-b\right), \\
u_{1}^{*}=\min \left(1, \max \left(0, \frac{1}{A_{1}}\left(\lambda_{2}^{*}-\lambda_{1}^{*}\right) \frac{\beta v_{I}^{*}(t) x^{*}(t)}{1+a v_{I}^{*}(t)}\right)\right), \\
\left.u_{2}^{*}=\min \left(1, \max \left(0, \frac{1}{A_{2}}\left(\lambda_{3}^{*}-\lambda_{4}^{*}\right) k y^{*}(t)\right)\right)\right), \\
\lambda_{i}\left(t_{f}\right)=0, \quad i=1, \ldots, 5 .
\end{gathered}
$$

\section{Numerical simulations}

In order to solve numerically our optimization system, we propose a numerical scheme based on forward and backward finite difference approximation. Then, we obtain the following algorithm.

\section{Numerical Algorithm.}

Initialization : $x_{0}, v_{I_{0}}, v_{N I_{0}}, y_{0}$ and $z_{0}$ are given. $u_{1}^{0}=0, u_{2}^{0}=0$ $\alpha_{N}^{1}=0, \alpha_{N}^{2}=0, \alpha_{N}^{3}=0, \alpha_{N}^{4}=0, \alpha_{N}^{5}=0$.

Iteration : Using the following steps, to compute solution:

step 1. For $i=0, \cdots, N-1$, do:

$$
\begin{aligned}
& x_{i+1}=x_{i}+\Delta h\left[\lambda-d x_{i}-\left(1-u_{1}^{i}\right) \frac{\beta v_{I_{i}} x_{i}}{1+a v_{I_{i}}}+r y_{i}\right], \\
& y_{i+1}=y_{i}+\Delta h\left[\left(1-u_{1}^{i}\right) \frac{\beta v_{I_{i}} x_{i}}{1+a v_{I_{i}}}-(\delta+r) y_{i}-p y_{i} z_{i}\right], \\
& v_{I_{i+1}}=v_{I_{i}}+\Delta h\left[\left(1-u_{2}^{i}\right) k y_{i}-\mu v_{I_{i}}\right], \\
& v_{N I_{i+1}}=v_{N I_{i}}+\Delta h\left[u_{2}^{i} k y_{i}-\mu v_{N I_{i}}\right] \\
& z_{i+1}=z_{i}+\Delta h\left[\frac{c y_{i} z_{i}}{1+\alpha y_{i}}-b z(i)\right] .
\end{aligned}
$$


step 2. Update the Lagrange multipliers:

$$
\begin{aligned}
& \alpha_{N-i-1}^{1}=\alpha_{N-i}^{1}-\Delta h\left[1+\alpha_{N-i}^{1} d\right. \\
& \left.+\left(\alpha_{N-i}^{1}-\alpha_{N-i}^{2}\right)\left(1-u_{1}^{i}\right) \frac{\beta v_{I_{i+1}}}{1+a v_{I_{i+1}}}\right], \\
& \alpha_{N-i-1}^{2}=\alpha_{N-i}^{2}-\Delta h\left[-\alpha_{N-i}^{1} r+\alpha_{N-i}^{2}\left(\delta+r+p z_{i+1}\right)\right. \\
& \left.-\alpha_{N-i}^{3}\left(1-u_{i}^{2}\right) k-\alpha_{N-i}^{4} u_{i}^{2} k-\alpha_{N-i}^{5} \frac{c z_{i+1}}{\left(1+\alpha y_{i+1}\right)^{2}}\right] \text {, } \\
& \alpha_{N-i-1}^{3}=\alpha_{N-i}^{3}-\Delta h\left[\left(\alpha_{N-i}^{1}-\alpha_{N-i}^{2}\right)\left(1-u_{i}^{1}\right) \frac{\beta x_{i+1}}{\left(1+a v_{I_{i+1}}\right)^{2}}+\alpha_{N-i}^{3} \mu\right] \text {, } \\
& \alpha_{N-i-1}^{4}=\alpha_{N-i}^{4}-\Delta h \alpha_{N-i}^{4} \mu \\
& \alpha_{N-i-1}^{5}=\alpha_{N-i}^{5}-\Delta h\left[1+\alpha_{N-i}^{2} p y_{i+1}-\alpha_{N-i}^{5}\left(\frac{c y_{i+1}}{1+\alpha y_{i+1}}-b\right)\right]
\end{aligned}
$$

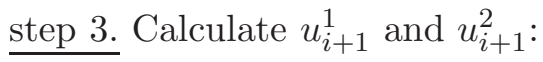

$$
\begin{gathered}
u_{i+1}^{1}=\min \left(1, \max \left(0, \frac{1}{A_{1}}\left(\alpha_{N-i-1}^{2}-\alpha_{N-i-1}^{1}\right) \frac{\beta v_{I_{i+1}} x_{i+1}}{1+a v_{I_{i+1}}}\right)\right), \\
\left.\left.u_{i+1}^{2}=\min \left(1, \max \left(0, \frac{1}{A_{2}}\left(\alpha_{N-i-1}^{3}-\alpha_{N-i-1}^{4}\right) k y_{i+1}\right)\right)\right)\right)
\end{gathered}
$$

end for

step 4. For $i=0, \cdots, N$, write

$$
\begin{gathered}
x^{*}(i)=x_{i}, y^{*}(i)=y_{i}, v_{I}^{*}(i)=v_{I_{i}}, v_{N I}^{*}(i)=v_{N I_{i}}, z^{*}(i)=z_{i}, \\
u_{1}^{*}(i)=u_{i}^{1}, \quad u_{2}^{*}(i)=u_{i}^{2} .
\end{gathered}
$$

end for

For our numerical simulations, we will choose the following parameters: $d=0.02 \mathrm{day}^{-1}, \beta=0.000024 \mathrm{~mm}^{3} \mathrm{day}^{-1}, \alpha=0.001, \lambda=5 \mathrm{day}^{-1} \mathrm{~mm}^{-1}, a=$ 0.001 day $^{-1}, p=0.001 \mathrm{~mm}^{3}$ day $^{-1}, \mu=3, k=330$ day $^{-1}, c=0.03 \mathrm{~mm}^{3}$ day $^{-1}$, $b=0.2$ day $^{-1}, A_{1}=250, A_{2}=2500$ and $r=0.01$ day $^{-1}$.

Moreover, besides the parameters we use the following initial conditions:

$$
x_{0}=190, v_{I 0}=1000, v_{N I 0}=100, y_{0}=2, z_{0}=10 .
$$

This complete data set was taken from $[11,30,12,31,32]$ and references therein. 


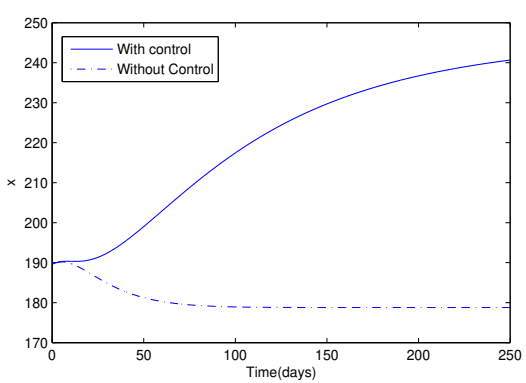

Figure 1: The evolution of the uninfected cells during time.

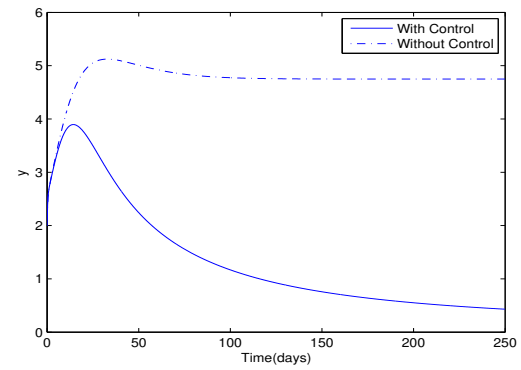

Figure 2: The evolution of the infected cells during time.

The graphs from simulating the model, given above, help to understand the effect of treatments with controls over the uninfected cells $(x)$, the infected cells $(y)$, the viral load $(v)$. In Figure 1, we notice that in the absence of treatment the $x$ cell count increases slowly the first ten days with considerable decrease. Whereas, in the presence of treatment, the $(x)$ population grows significantly, which improves the quality of life of the patient. In Figure 2, we remark that before the treatment, the infected cells increase quickly during the first ten days with light decrease. While on the contrary, after the treatment, the number of infected cells dropped importantly. The curve representing the infected cells under control converges toward 0.52 , nevertheless, without control, it converges towards 4.85. This proves the role of control in reducing the number of infected cells, which contributes to curing the disease. In figure 3, we show that after introducing therapy, the viral load declines towards zero, whereas, without control, it stays equal to 585.11. Finally, figure 4 represents the optimal controls $u_{1}$ and $u_{2}$ in blocking new infection and inhibiting viral production. The plots show that a patient should take the two treatments during the first 


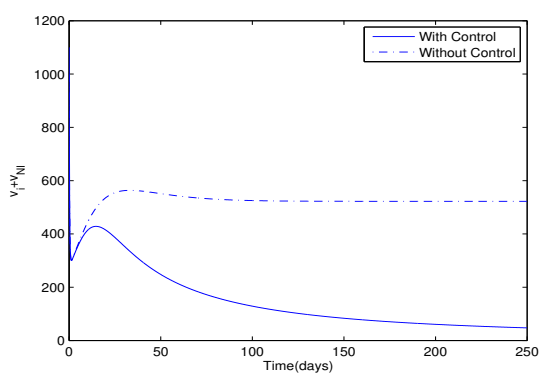

Figure 3: The evolution of the HIV virus $\left(v=v_{I}+v_{N I}\right)$ during time.
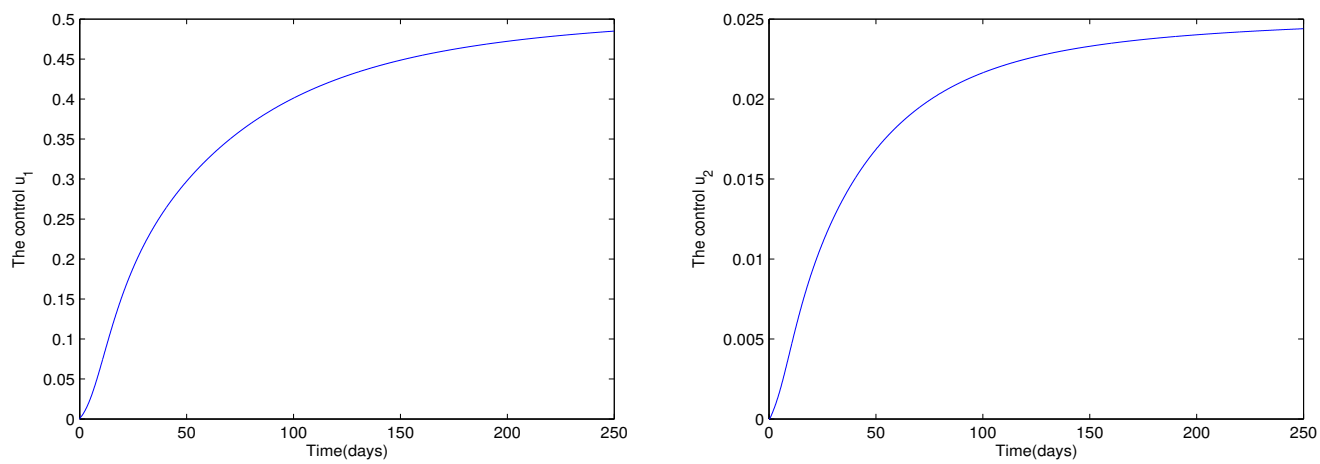

Figure 4: The behaviour of the optimal controls.

days of therapy in an increasing manner and both of the two treatments should be administrated for the lifetime period in a constant way.

\section{Summary and conclusion}

In this work, we have studied a new mathematical model that describes the human immunodeficiency virus with the CTL response, two saturated rate with inclusion of two drug combinations. Optimal control theory is applied to determine the optimal treatment regime. The study applied Pontryagins Maximum Principle in deriving the conditions for optimal control, which maximizes the objective function. The systems of ODEs, the state system and the adjoint system were solved numerically. The numerical results show that the optimal treatment strategies reduce viral load and increase the uninfected cell count 
after some days of therapy. In the future, it is important to study the dynamics of such model.

\section{References}

[1] R.A. Weiss, How does HIV cause AIDS?, Science, 260 (1993), 1273-1279.

[2] W. Blanttner, R.C. Gallo, H.M. Temin, HIV causes aids, Science, 241 (1988), 515-516.

[3] C.J. Silva, D.F.M. Torres, A TB/AIDS coinfection model and optimal control treatment, Discrete and continuous dynamical systems, $\mathbf{3 5}$, No 9 (2015), 4639-4663.

[4] Panel on Antiretroviral Guidelines for Adults and Adolescents. Guidelines for the use of antiretroviral agents in HIV-1-infected adults and adolescents, Department of Health and Human Services (2011), 1-166.

[5] M.A. Nowak, R.M. May, Mathematical biology of HIV infection: antigenic variation and diversity threshold, Math. Biosci, 106, No 1 (1991), 1-21.

[6] M.A. Nowak, C.R.M. Bangham, Population dynamics of immune responses to persitent viruses, Science, 272 (1996), 7479.

[7] A. Perelson, A. Neumann, M. Markowitz, J. Leonard, D. Ho, HIV-1 dynamics in vivo: Virion clearance rate, infected cell life-span, and viral generation time, Science, 271 (1996), 582-1586.

[8] W. Liu, Nonlinear oscillation in models of immune response to persistent viruses, Theor. Popul. Biol., 52 (1997), 224230.

[9] A.S. Perelson, P. W. Nelson, Mathematical analysis of HIV-1 dynamics in vivo, SIAM Rev., 41, No 1 (1999), 3-44.

[10] M.A. Nowak, R. M. May, Viral Dynamics, Oxford University Press, Oxford (2000).

[11] S.M. Ciupe, B. Bivort, D. Bortz, P. Nelson, Estimates of kinetic parameters from HIV patient data during primary infection through the eyes of three different models, Math. Biosci., 200 (2006), 1-27.

[12] K. Hattaf and N. Yousfi, Dynamics of HIV infection model with therapy and cure rate, Inter. J. of Tomography and Statistics, 16 (2011), 74-80. 
[13] B. El Boukari, K. Hattaf, N. Yousfi, Modeling the therapy of HIV infection with CTL response and cure rate, Int. J. Ecol. Econ. Stat., 28 (2013), 117.

[14] Y. Tabit, K. Hattaf, N. Yousfi, Dynamics of an HIV pathogenesis model with CTL immune response and two saturated rates, World J. of Modelling and Simulation, 10, No 3 (2014), 215223.

[15] Q. Sun, L. Min, Dynamics Analysis and Simulation of a Modified HIV Infection Model with a Saturated Infection Rate, Computational and Mathematical Methods in Medicine, 2014 (2014), 145-162.

[16] Y. Tabit, A. Meskaf, K. Allali, Mathematical analysis of HIV model with two saturated rates, CTL and antibody responses, World J. of Modelling and Simulation, 12, No 2 (2016), 137-146.

[17] K. Allali, Y. Tabit and S. Harroudi, On HIV model with adaptive immune response, two saturated rates and therapy, Mathematical Modelling of Natural Phenomena, 12, No 5 (2017), 1-14.

[18] X. Zhou, X. Song, X. Shi, A differential equation model of HIV infection of CD4+ T-cells with cure rate, J. Math. Anal. Appl., 342, No 2 (2008), 1342-1355.

[19] X. Liu, H. Wang, W. Ma, Global stability of an HIV pathogenesis model with cure rate, Nonlinear Anal. RWA, 12, No 6 (2011), 2947-2961.

[20] X. Song, A. Neumann, Global stability and periodic solution of the viral dynamics, J. Math. Anal. Appl., 329, No 1 (2007), 281-297.

[21] S. Iwami, T. Miura, S. Nakaoka, Y. Takeuchi, Immune impairment in HIV infection: Existence of risky and immunodeficiency thresholds, J. of Theoretical Biology, 260, No 4 (2009), 490-501.

[22] K. Hattaf, N. Yousfi, Two optimal treatment of HIV infection medel, World J. of Modelling and Simualtion, 8, No 1 (2012), 27-35.

[23] D. Rocha, C.J. Silva, D.F.M. Torres, Stability and optimal control of a delayed HIV model, Mathematical Methods in Appl. Sci. (2016).

[24] A. Meskaf, K. Allali, Y. Tabit, Optimal control of a delayed hepatitis B viral infection model with cytotoxic T-lymphocyte and antibody responses, Intern. J. of Dynamics and Control, 5 (2017), 893-902. 
[25] S. Harroudi, D. Bentaleb, Y. Tabit, S. Amine, K. Allali, Optimal control of an HIV infection model with the adaptive immune response and two saturated rates, International Journal of Mathematics and Computer Science, 14 (2019), 787807.

[26] H.R. Thieme, Mathematics in Population Biology, Princeton University Press, Princeton (2003).

[27] W.H. Fleming, R.W. Rishel, Deterministic and Stochastic Optimal Control, Springer (1975).

[28] D. L. Lukes, Differential Equations: Classical to Controlled, Vol. 162 of Mathematics in Science and Engineering, Academic Press (1982).

[29] L.S. Pontryagin, V.G. Boltyanskii, R.V. Gamkrelidze, E.F. Mishchenko, The Mathematical Theory of Optimal Processes, Wiley (1962).

[30] S. Bonhoeffer, M. Rembiszewski, G.M. Ortiz and D.F. Nixon, Risks and benefits of structured antiretroviral drug therapy interruptions in HIV-1 infection, AIDS, 14 (2000), 2313-2322.

[31] K. Hattaf and N. Yousfi, A delay differential equation model of HIV with therapy and cure rate, Intern. J. of Nonlin. Sci., 12 (2011), 503512.

[32] H. Zhu and X. Zou, Dynamics of a HIV-1 infection model with cellmediated immune response and intracellular delay, Discrete and Continuous Dynam. Systems Ser. B, 12, No 2 (2009), 511-524. 
